\title{
EBV-LMP1-targeted DNAzyme induces DNA damage and causes cell cycle arrest in LMP1-positive nasopharyngeal carcinoma cells
}

\author{
XIAOQIAN MA ${ }^{1,3^{*}}$, ZHIJIE XU ${ }^{1,2^{*}}$, LIFANG YANG ${ }^{1,2}$, LANBO XIAO $^{1,2}$, MIN TANG ${ }^{1,2}$, JINGCHEN LU ${ }^{1,2}$, \\ SAN XU ${ }^{1,2}$, YIPING TANG ${ }^{1,2}$, XINXIAN WEN ${ }^{1,2}$, XINGMING DENG $^{5}$, LUNQUAN SUN ${ }^{4}$ and YA CAO ${ }^{1,2}$ \\ ${ }^{1}$ Cancer Research Institute, ${ }^{2}$ Key Laboratory of Carcinogenesis and Cancer Invasion of Ministry of Education, \\ Xiangya School of Medicine, Central South University, Changsha, Hunan 410078; ${ }^{3}$ Cell Transplantation and \\ Gene Therapy Institute, The 3rd Xiangya Hospital, Central South University, Changsha, Hunan 410013; \\ ${ }^{4}$ Center for Molecular Medicine, Xiangya Hospital, Central South University, Changsha, Hunan 410078, P.R. China; \\ ${ }^{5}$ Department of Radiation Oncology, Emory University School of Medicine and \\ Winship Cancer Institute of Emory University, Atlanta, GA 30322, USA
}

Received July 19, 2013; Accepted August 23, 2013

DOI: $10.3892 /$ ijo.2013.2098

\begin{abstract}
This study aimed to determine the molecular mechanisms underlying the effect of the LMP1-targeted DNAzyme 1 (DZ1) on cell cycle progression in nasopharyngeal carcinoma (NPC) cells. We showed that the active DZ1 inhibited the expression of latent membrane protein 1 (LMP1) and induced a G1 phase arrest. In addition, this cell cycle deregulation was shown to be accompanied by upregulation of the DNA damage marker $\gamma$-H2AX, downregulation of the DNA damage response factor p-p53-Ser15 and cell proliferation inhibition. To investigate what affected the cell cycle progression, we examined the expression of two checkpoint-related cyclins and cyclin-dependent kinases (CDKs). We found a decrease of cyclin D1 and cyclin E protein levels at $24 \mathrm{~h}$ from the DZ1 treatment. Moreover, we observed inhibition of CDK4 activity and decreased cyclin D1 expression in the complexes immunoprecipitated with CDK4 antibody. We also found a reduction in cdc 2 phosphorylation at Thr161 which partially stands for the cdc2 kinase activity in DZ1-treated CNE1-LMP1 cells, although the downregulation of LMP1 expression had no effect on the cyclin B1 and cdc2
\end{abstract}

Correspondence to: Dr Lunquan Sun, Center for Molecular Medicine, Xiangya Hospital, Central South University, Changsha, Hunan 410078, P.R. China

E-mail: lq-sun@hotmail.com

Dr Ya Cao, Cancer Research Institute, Xiangya School of Medicine, Central South University, Changsha, Hunan 410078, P.R. China

E-mail:ycao98@vip.sina.com

*Contributed equally

Key words: cell cycle, latent membrane protein 1, DNAzyme, nasopharyngeal carcinoma expression. Further, we analyzed changes in cdc2 kinase activity induced by DZ1 and found that the downregulation of the LMP1 expression resulted in a 5-fold reduction in cdc2 kinase activity in CNE1-LMP1. The data suggest that the downregulation of the LMP1 expression by DZ1 was able to induce DNA damage, which then further inhibited the cell proliferation and resulted in malfunction of cell cycle checkpoints that led to G1 phase arrest and the decrease in number of cells in $\mathrm{G} 2 / \mathrm{M}$ phase.

\section{Introduction}

Epstein-Barr virus (EBV) is a human $\gamma$ herpesvirus commonly carried in the majority of the human population. It is implicated in a variety of human malignancies that include Burkitt's lymphoma, nasopharyngeal carcinoma, Hodgkin's lymphoma, nasal $\mathrm{T}$ cell lymphoma, and immunoblastic lymphomas in post-transplant and AIDS patients. EBV-encoded LMP1 is the only one implicated in cell immortalization and transformation. It is required for B-cell immortalization, together with the EBNA2, EBNA3a and EBNA3c genes (1-4). In addition, LMP1 has been shown to induce the transformation of certain established rodent fibroblast cell lines, including Rat-1 and BALB/c 3T3 (5-7). Furthermore, LMP1 induces the tumorigenicity of epithelial cell lines in severe combined immunodeficient mice $(8,9)$.

As a $60 \mathrm{kDa}$ integral membrane protein, LMP1 functions as a constitutively active tumor necrosis factor receptor (TNFR) and, in certain instances, can substitute for CD40 in vivo, providing both growth and differentiation responses in B lymphocytes (10). LMP1 also contributes to multiple aspects of NPC development through activating a number of signaling pathways including nuclear factor $\mathrm{NF}-\kappa \mathrm{B}$, activator protein-1 (AP-1), Janus kinase/signal transducer and activator of transcription (JAK/STAT) (11-14). Activation of NF- $\kappa$ B or AP-1 by LMP1 has been linked to the upregulation of some cellular proteins and inhibition of apoptosis. LMP1 has clear oncogenic 
properties in multiple cell backgrounds and in transgenic mice. In NPCs, LMP1 expression is heterogeneous and associated with metastasis (15), supported by studies in epithelial cultures showing LMP1 induced changes in cell morphology, adhesion, cell motility and exo-proteolytic activity (16). Like many classical oncogenes, the growth promoting properties of LMP1 appear to be cell-type specific. Whereas LMP1 expression promotes growth and proliferation in rodent fibroblasts and certain human epithelial cell lines such as C33A and HONE1, its expression in RHEK-1, SVK and Hep-G2 cells is associated with growth inhibition and, under certain circumstances, cytostasis or apoptosis (17).

Interference with LMP1 function eliminates tumorigenic potential. Recently there have been some reports that the RNA interference against LMP1 exhibited anti-proliferative and anti-metastasis effects in the LMP1 expressing NPCs $(18,19)$. Downregulation of the LMP1 expression has been shown to induce apoptosis, and sensitize the EBV-positive cells to cytotoxic agents $(20,21)$. Although the biological effects of inhibition of LMP1 expression has been well demonstrated, its cell biological basis is yet to be elucidated. In a previous study we demonstrated that the '10-23' DNAzymes specifically targeting the LMP1 mRNA significantly downregulated the expression of LMP1 in the nasopharyngeal carcinoma cell line CNE1-LMP1 which led to sensitization of LMP1 positive cells to radiation (22). In this study we found that after DNAzyme 1 treatment the CNE1-LMP1 cells accumulated in G1 phase and the percentage of cells in $\mathrm{G} 2 / \mathrm{M}$ phase was decreased. This cell cycle change was accompanied by $\gamma-\mathrm{H} 2 \mathrm{AX}$, a DNA damage marker, increased and the phospharylation of p53 decreased. However, the mechanisms of the cell cycle arrest caused by downregulation of LMP1 are unclear. In the present study, we attempt to examine the expression feature of the molecules associated with the cell cycle control in G1/S and G2/M checkpoints, following the silencing of the LMP1 expression by the LMP1-targeted DNAzyme 1. We show that both checkpoints contributed to the G1 phase arrest and cells in G2/M phase decreased induced by the downregulation of LMP1. Our results thus suggest that LMP1 is an important regulator of the cell cycle in LMP1-positive NPC cells.

\section{Materials and methods}

Cell culture. CNE1 is an LMP1 negative, low differentiated nasopharyngeal squamous carcinoma cell line. CNE1-LMP1 is a cell line transfected with EBV-LMP1 (23) and shown to have accelerated cell proliferation (24). The cells were cultured in RPMI-1640 medium supplemented with $10 \%$ fetal bovine serum (FBS, Gibco), $100 \mathrm{IU} / \mathrm{ml}$ penicillin, $100 \mathrm{mg} / \mathrm{ml}$ streptomycin, and $2 \mathrm{mM} / \mathrm{l} \mathrm{L}$-glutamine in a humidified atmosphere of $5 \% \mathrm{CO}_{2}$ at $37^{\circ} \mathrm{C}$.

DNAzyme transfection of CNE1-LMP1 or CNE1 cells. Briefly, the ' $10-23$ ' model was adopted by incorporating $9 \mathrm{bp}$ arms at each side of the catalytic motif. To increase stability of DNAzymes in cells, two phosphorothioate linkages were introduced to both ends of the arms. A DNAzyme oligonucleotide control (ODN) was a scrambled sequence that consists of the same nucleotide composition. To transfect cells with DNAzymes, tetra (4-methyridyl) porphyrine (TMP) was used to facilitate the cellular uptake of the catalytic oligonucleotides (25). Prior to transfection, cells were seeded in 6-well plates overnight. The DNAzymes/TMP mixtures were made at a charge ratio (C/R) of 1 with $2 \mu \mathrm{M}$ DNAzyme oligonucleotides as described (26). The mixtures were incubated for $15 \mathrm{~min}$ at room temperature to form the transfection complex. After the cells were rinsed twice with PBS, the transfection mixture was added to the cells and incubated at $37^{\circ} \mathrm{C}$ for $4 \mathrm{~h}$ in $5 \% \mathrm{CO}_{2}$, followed by the addition of complete medium to the wells and further incubation for the indicated time.

Cell cycle distribution analysis. Flow cytometry analysis of PI-stained cells was performed to study the effect of the active DNAzyme on the cellular cycle. Cells $\left(1 \times 10^{6}\right)$ were cultured with or without $2 \mu \mathrm{M}$ of DNAzyme and collected $24 \mathrm{~h}$ later. Treated and untreated cells were then washed with ice-cold PBS and suspended in $75 \%$ ethanol at $-20^{\circ} \mathrm{C}$ overnight. Fixed cells were centrifuged and washed with PBS twice. Before flow cytometry analysis, cells were stained with $50 \mu \mathrm{g} / \mathrm{ml}$ of propidium iodide and $0.1 \%$ of RNase A in $400 \mu \mathrm{l}$ PBS in a lightproof tube at $25^{\circ} \mathrm{C}$ for $30 \mathrm{~min}$. Stained cells were assayed on FACSort (Becton-Dickinson) and the cell cycle parameters and the percentage of apoptotic cells (sub-G1 peak) were determined using the CellQuest software program (Becton-Dickinson).

Western blot analysis. Cells grown in 6-well plates were cultured without or with DNAzymes. Cells were harvested at different time points, washed with ice-cold PBS and lysed in the lysis buffer (10 mM Tris-HCl, pH 8.0, 1 mM EDTA, 2\% SDS, $5 \mathrm{mM}$ DTT, $10 \mathrm{mM}$ PMSF, proteinase inhibitor mix) for $30 \mathrm{~min}$ on ice. The lysate was centrifuged for $15 \mathrm{~min}$ at $13,000 \mathrm{rpm}$ in a microfuge at $4{ }^{\circ} \mathrm{C}$. After protein quantification by the BCA Assay Reagent (Pierce Chemical, Inc), $50 \mu \mathrm{g}$ of the total proteins from the treated or untreated cells were mixed with the sample buffer and boiled for $5 \mathrm{~min}$. The samples were then resolved on a $10 \%$ polyacrylamide SDS gel and transferred onto a nitrocellulose membrane by electroblotting. The membrane was incubated in blocking buffer (TBS containing 5\% skimed milk and $0.1 \%$ Tween-20) for $2 \mathrm{~h}$, followed by incubation with primary antibody diluted in the same buffer $(1: 1,000)$. The membrane was washed in TBS containing $0.1 \%$ Tween-20 and further incubated with a secondary antibody that could be detected using a peroxidase-conjugated anti-IgG at 1:10,000. Protein expression were determined using a supersignal chemiluminescence system (ECL, Pierce) followed by exposure to autoradiographic film. The antibodies used in the study include LMP1 (Dako), mouse monoclonal anti- $\beta$-actin (Sigma), mouse monoclonal anti- $\alpha$-tublin (Santa Cruz), rabbit anti-phosphoserine (Zymed), rabbit polyclonal anti-cdc2 p34 (Santa Cruz), rabbit polyclonal anti-phospho-Thr161-cdc2 and rabbit polyclonal anti-phospho-Tyr15-cdc2 (Cell Signaling), mouse monoclonal anti-cyclin B1 (Santa Cruz), p-p53 (Ser15) (1:500, Santa Cruz), and $\gamma$-H2AX (1:1,000, Millipore). Secondary antibodies were horseradish peroxidase (HRP)-conjugated rabbit anti-mouse IgG (Santa Cruz), HRP-conjugated goat anti-rabbit IgG (Santa Cruz).

Immunofluorescent analysis. CNE1 and CNE1-LMP1 cell lines were seeded on Millicell EZ slides (Millipore), and subjected to the following treatments: untreated, DNAzyme- or control ODN-treated for $24 \mathrm{~h}$. Cells were fixed in ice cold methanol for 
10 min and washed with PBS, then blocked with 5\% donkey serum/PBS for $1 \mathrm{~h}$. The cells were subsequently incubated with the mouse anti- $\gamma$-H2AX (1:450, Millipore) overnight. Followed by washing with PBS for $15 \mathrm{~min}$, AlexaFluor 488-conjugated goat anti-mouse secondary antibodies (1:1,000, Molecular Probes, Eugene, OR) were used. All samples were mounted in Prolong Gold antifade reagent with DAPI (Molecular Probes) and fluorescence images were captured using Leica DMI3000 B (Leica, Germany).

Immunoprecipitation kinase assay. The cyclin D1-CDK4 kinase assay was performed as described with modifications (27). Cells were transfected with LMP1-specific DNAzyme 1 or control ODN $24 \mathrm{~h}$ after the transfection, cells were washed twice with ice-cold PBS and harvested with a kinase buffer [25 mM Tris- $\mathrm{HCl}(\mathrm{pH} 7.5), 5 \mathrm{mM}$ glycerophosphate, $2 \mathrm{mM}$ DTT, $0.1 \mathrm{mM} \mathrm{Na} \mathrm{VO}_{4}, 10 \mathrm{mM} \mathrm{MgCl}$, and $1 \mathrm{mM}$ PMSF]. Protein content was determined using the BCA assay. Cyclin D1 was immunoprecipitated from $600 \mu \mathrm{g}$ of the cell extract using an anti-cyclin D1 monoclonal antibody diluted at 1:100 overnight. A total of $20 \mu \mathrm{l}$ of protein $\mathrm{A} / \mathrm{G}$ PLUS-Agarose (Santa Cruz) was added, incubated at $4^{\circ} \mathrm{C}$, and then washed at $4^{\circ} \mathrm{C}$ four times with ice-cold kinase buffer. Immunoprecipitated cyclin D1 was incubated with $1 \mu \mathrm{g} \mathrm{Rb}-\mathrm{C}$ fusion protein (Cell Signaling) and $200 \mu \mathrm{M}$ ATP in a kinase buffer at $30^{\circ} \mathrm{C}$ for $30 \mathrm{~min}$. Phosphorylation of $\mathrm{Rb}-\mathrm{C}$ fusion protein was detected by western blot analysis with antiphospho-Rb (Ser780) polyclonal antibodies.

cdc2 Kinase Activity Assay. The cdc2 kinase activity assay was carried out according to the instruction in the MESACUP Cdc2 Kinase Assay Kit. Cells were lysed in a sample buffer (50 mM Tris- $\mathrm{HCl}$, pH 7.5, 0.5 M NaCl, $5 \mathrm{mM}$ EDTA, $2 \mathrm{mM}$ EGTA, 0.01\% Brij35, $1 \mathrm{mM}$ PMSF, $0.05 \mathrm{mg} / \mathrm{ml}$ leupeptin, $50 \mathrm{mM} \beta$-glycerophosphate, $1 \mathrm{mM}$ Na-orthovanadate). After sonication, cell extracts were separated. The amount of $600 \mu \mathrm{g}$ cell extracts were incubated with HCK-gel suspension (MBL, Code no. 5236) on ice for $1 \mathrm{~h}$. Followed by centrifugation, the pellets were separated and used for an enzyme source. Reaction reagents added into the HCK-gel pellets included $10 \mathrm{x}$ cdc 2 reaction buffer, biotinylated MV peptide, ATP and distilled water. Following incubation for $30 \mathrm{~min}$ with the sample buffer as a control, the phosphorylation reaction was terminated to permit the cdc 2 kinase activity to be measured. The reaction mixture was transferred to a microwell strip coated with monoclonal antibody (4A4), incubated at $25^{\circ} \mathrm{C}$ for $60 \mathrm{~min}$, and washed with the washing solution. Peroxidase (POD)-conjugated streptavidin was added and incubated, and then the mixture was washed once again. POD substrate solution was added and incubated for 3 to $5 \mathrm{~min}$. Stop solution was then added, and the optical density (OD) of each well was read at $490 \mathrm{~nm}$ with a microplate reader.

Co-immunoprecipitation analysis (Co-IP). CDK4 was pulled down by anti-CDK4 antibody, and then precipitated by binding with protein A Sepharose CL-4B beads (Pharmacia, USA). Anti-cyclin D1 antibody was used as the primary antibody to detect the levels of cyclin D1. A co-immunoprecipitation (Co-IP)/ western blot assay was performed to analyze the interaction of CDK4 with cyclin D1. The cell extract was prepared for immu- noprecipitation or Co-IP/western blot analysis. The harvested cells were lysed in IP lysis buffer (50 mM Tris- $\mathrm{HCl}, 150 \mathrm{mM}$ $\mathrm{NaCl}, 10 \%$ Nonidet P-40, 1 mM EDTA, 10\% glycerol, $10 \mathrm{mM}$ $\mathrm{NaF}, 1 \mathrm{mM} \mathrm{Na} \mathrm{VO}_{4}, 1 \mathrm{mM}$ DTT, $1 \mathrm{mM}$ PMSF, and phosphatase inhibitor cocktail). The supernatant was mixed with protein $\mathrm{A}$ Sepharose CL-4B beads, incubated for $2 \mathrm{~h}$, centrifuged for $2 \mathrm{~min}$ at 2,000 rpm for pre-clearing and incubated overnight with CDK4 antibody and protein A Sepharose CL-4B beads, followed by centrifugation for $2 \mathrm{~min}$ at $12,000 \mathrm{rpm}$. The immunoprecipitates were collected, washed five times, and finally subjected to western blot analysis.

Co-IP was used to analyze protein interaction with a special antibody linked to protein A Sepharose CL-4B beads (Pharmacia). Immunoprecipitates were separated for western blot analysis.

MTS analysis. MTS assays were performed to assess the effect of DNAzymes on cell proliferation. Briefly, $200 \mu \mathrm{l}$ of logarithmically growing cells were plated on a 96-well plate and LMP1-targeted-DNAzyme 1 or control ODN were transfected as described above. Then cells were incubated at $37^{\circ} \mathrm{C}$ and $100 \mu \mathrm{l}$ of sterile MTS dye (30156802, Promega) were added to each well at different time points. The plates were further incubation at $37^{\circ} \mathrm{C}$ for $4 \mathrm{~h}$. After that, spectrometric absorbance at the wavelength of $570 \mathrm{~nm}$ was measured on a microplate reader (Bio-Tek Inc). The background absorbance of medium in the absence of cells was subtracted. All samples were assayed in triplicate, and the mean for two experiments was used to calculate cell proliferation rate expressed as percentage of control.

Statistical analyses. All data were shown as mean \pm standard deviation. The difference between 2 groups of data was examined by Student's t-test. The statistical difference at $\mathrm{p}<0.05$ was considered as significantly and $p<0.01$ as very significant.

\section{Results}

EBV-LMPl-targeted DNAzyme 1 inhibits the expression of EBV-LMPI and affects the cell cycle distribution in CNE1-LMP1 cells. In order to determine if the DNAzyme 1 could affect the target gene expression, we used a cationic porphyrin TMP, as a transfection reagent, for transfecting DNAzyme oligonucleotides into CNE1-LMP1 cells. DZ1 $(2 \mu \mathrm{M})$ was transfected into both CNE1-LMP1 cells and CNE1 cells (as a negative target control). The effect on the LMP1 expression at the protein level was assayed using western blot analysis. As shown in Fig. 1A, DZ1 significantly inhibited the LMP1 protein expression in CNE1-LMP1 cells, while the control ODN had no effect on the LMP1 expression.

Since LMP1 can promote cell growth and accelerate cell cycle progression, we next examined the effect of downregulation of LMP1 on cell cycle. DZ1 was transfected into CNE1-LMP1 and CNE1 cells after overnight serum starvation and the cell cycle distribution was assessed by flow cytometry, it was shown that DZ1 significantly increased the percentage of cells in G1 phase (49.06 \pm 3.12$)$ compared to the untreated $(33.83 \pm 4.96)$ or control ODN $(38.01 \pm 2.18)$ in LMP1-positive cells. This increase in the G1 phase was coupled with a significant decrease in the percentage of cells in $\mathrm{G} 2 / \mathrm{M}$ phase 
A
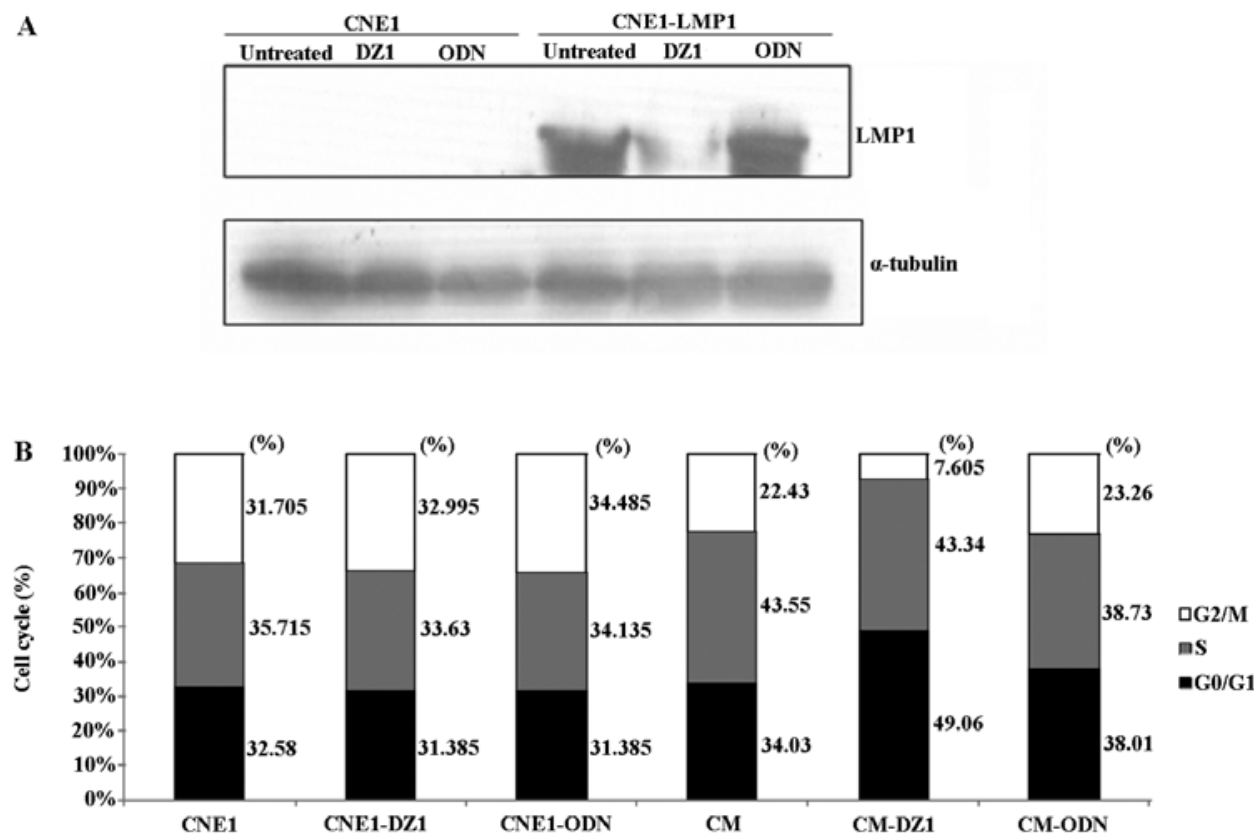

Figure 1. DZ1 inhibition of LMP1 protein expression in CNE1-LMP1 changing the cell cycle distribution (A) LMP1 was downregulated by DZ1. (B) G1 phase arrest caused by LMP1-targeted DNAzyme 1 in CNE1-LMP1 cells but not in CNE1 cells.

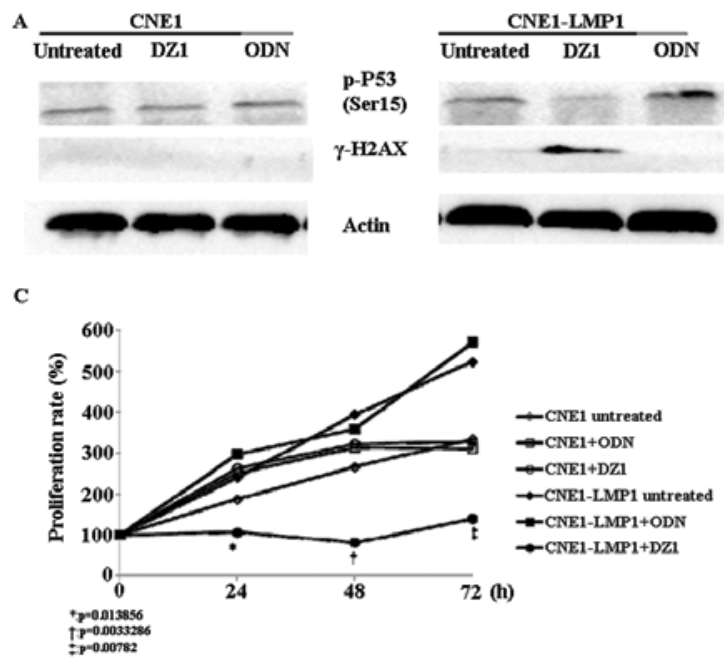

B
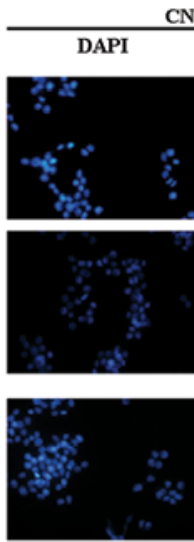

CNE1
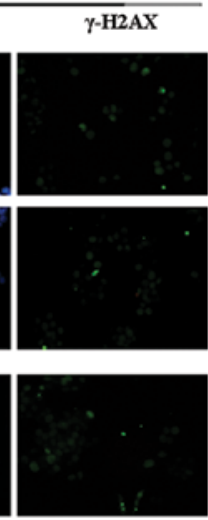

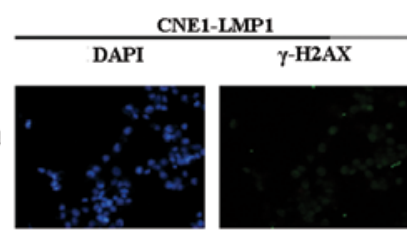

DZ1
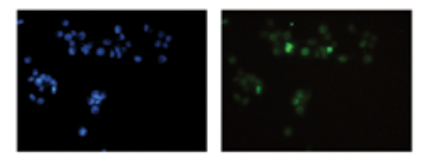

ODN
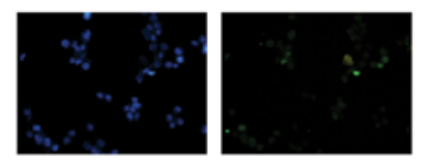

Figure 2. LMP1-targeted DZ1 decreased p-p53 (Ser15), increased $\gamma$-H2AX and inhibited cells proliferation in the CNE1-LMP1 cell lines. CNE1 and CNE1-LMP1 cells were transfected with DZ1 or control ODN $(2 \mu \mathrm{M})$ for $24 \mathrm{~h}$ and then harvested. (A) Total cell lysate was analyzed by western blotting using antibodies targeting p-p53 and $\gamma-\mathrm{H} 2 \mathrm{AX}$. $\beta$-actin was used for the loading control. (B) Immunofluorescent staining revealed that CNE1-LMP1 but not CNE1 transfected with LMP1-targeted DZ1 had significantly increased $\gamma$-H2AX foci. (C) MTS showed that DZ1 inhibited LMP1-positive cell proliferation from 24 to $72 \mathrm{~h}$.

(7.61 \pm 3.84 of DZ1 treated LMP1-positive cells vs $22.43 \pm 1.41$ of untreated or $23.26 \pm 1.85$ of control ODN treated LMP1-positive cells) after $24 \mathrm{~h}$ of transfection. Whereas, such an effect was not observed on the cell cycle of LMP1-negative tumor cell (CNE1) (Fig. 1B). These data confirmed that DZ1 could downregulate the LMP1 expression, resulting in G1 phase arrest of CNE1-LMP1 cells and a decreased cell number in G2/M phase.

EBV-LMP1-targeted DNAzyme 1 induces DNA damage in CNE1-LMP1 cells and inhibits cell proliferation. Since DNA damage is an early event of cell cycle arrest, we examined whether DZ1 could induce DNA damage in CNE1-LMP1 cells through inhibiting LMP1. As shown in Fig. 2A, the expression level of $\gamma-\mathrm{H} 2 \mathrm{AX}$, a marker of DNA damage, was significantly increased by DZ1 in CNE1-LMP1 cells compared with the untreated or control ODN treated cells. This result was also confirmed by immunofluorescence (Fig. 2B). When LMP1 was inhibited, the $\gamma-\mathrm{H} 2 \mathrm{AX}$ level was highly induced in CNE1-LMP1 cells.

It is known that p53 is required to promote the DNA damage induced response. One key event for p53-mediated DNA damage response is phosphorylation of serine 15 (Ser15) in human p53 (28). To evaluate whether the LMP1-targeted DNAzyme mediated the DNA damage response, we examined 


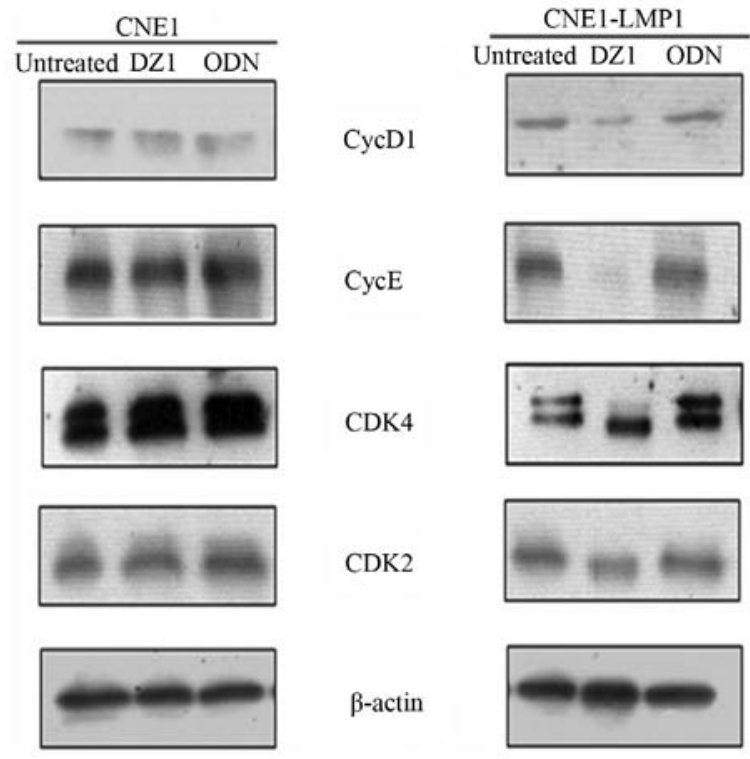

Figure 3.Effect of DZ1 on G1/S cell cycle regulators in CNE1 and CNE1-LMP1 cells. CNE1 and CNE1-LMP1 cells grown in 6-well plates were transfected with DZ1 or control ODN $(2 \mu \mathrm{M})$ for $24 \mathrm{~h}$. Total cell lysates were analyzed by western blotting using specific antibodies, respectively.

the level of p-p53 (Ser15) by western blot analysis. It was shown that the phosphorylation of Ser15 was downregulated in DZ1-treated CNE1-LMP1 cells (Fig. 2A).

To explore whether the LMP1-targeted DNAzyme 1 could affect cell proliferation, MTS was performed. The results showed that CNE1-LMP1 cells grew faster than CNE1 cells.
However, when CNE1-LMP1 is transfected with DZ1, they have a significant slower growth rate than the cells transfected with negative control or untreated cells from 24 to $72 \mathrm{~h}$ (Fig. 2C).

EBV-LMP1-targeted DNAzyme 1 affects the expression of the genes that regulate G1/S progression in CNE1-LMPI cells. Cells entering the proliferation phase go first through the G1/S checkpoint, which is mainly regulated by CDK4 and cyclin D1 and other CDKs and cyclins. We transfected the DZ1 to the CNE1-LMP1 and CNE1 cells, and examined the expression of the G1/S checkpoint-related genes. It was found that DZ1 markedly decreased the expression of CDK4 and cyclin D1, as well as CDK2 and cyclin E. These changes were not found in the LMP1 negative CNE1 cells (Fig. 3). The results suggested that DZ1 specifically inhibited the LMP1 expression resulting in the downregulation of the expression of the G1/S checkpoint related cyclins and CDKs.

It is necessary for CDKs and cyclins to interact directly to function in the cell cycle control. Western blot analysis demonstrated that inhibition of LMP1 expression led to downregulation of cyclin D1 and CDK4. To investigate if the effect was due to a weaker interaction between CDK4 and cyclin D1, Co-IP analysis was used to assay such an interaction. CDK4 was pulled down by excess anti-CDK4 antibody, a co-immunoprecipitation (Co-IP) assay performed to analyze cyclin D1. Analysis of total cell lysates showed that the DZ1-mediated inhibition of LMP1 resulted in a decrease of the level of CDK4 and also the associated cyclin D1, which suggested a decrease of the interaction between cyclin D1 and CDK4 (Fig. 4A).

$\mathrm{pRb}$ is a key regulator in the transition from $\mathrm{G} 1$ to $\mathrm{S}$ and its activity is modulated by cyclin D-CDK complexes.
A IB:

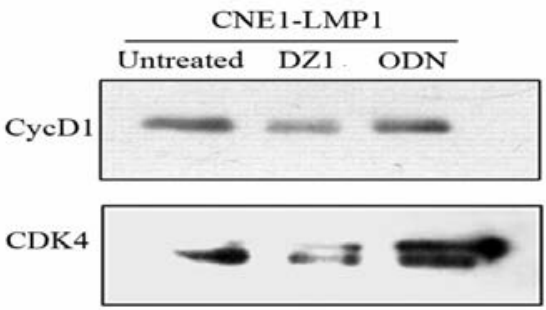

C

Untreated DZ1
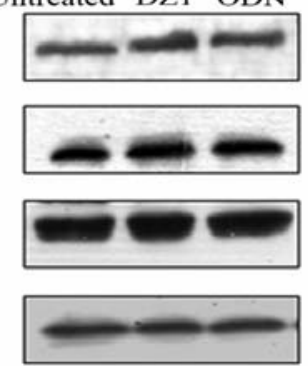

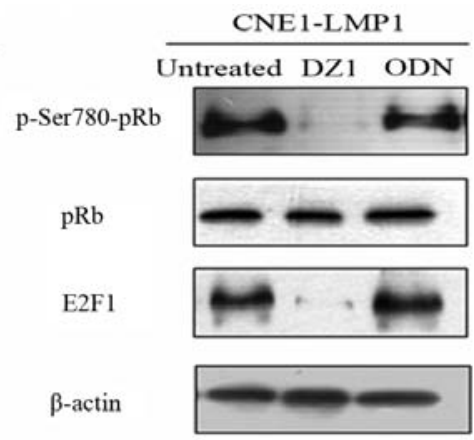

CNE1-LMP1

B

CNE1

CNE1-LMP1

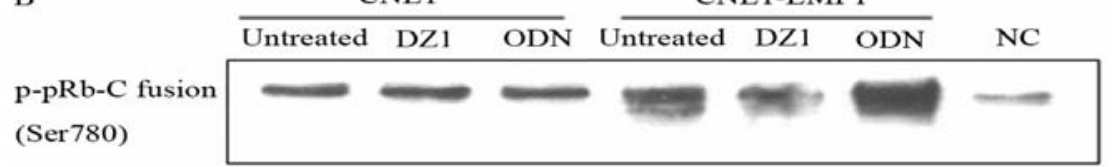

Figure 4. LMP1-targeted DNAzyme 1 mediated effect on CDK4/cyclin D1-pRb-E2F1 signaling pathways in CNE1-LMP1. (A) Analysis of the association between CDK4 and cyclin D1 in CNE1-LMP1 cells. Cell lysates from DZ1 or control ODN transfected CNE1-LMP1 cells and untreated cells were immunoprecipitated with an anti-CDK4 antibody. Lysates were immunoblotted with the corresponding antibodies as marked. (B) DZ1 suppression of the activation of the cyclin D1-CDK4 complex in CNE1-LMP1 cells. CNE1 and CNE1-LMP1 cells grown in 6-well plates were transfected with DNAzymes or control ODN (2 $\mu$ M) for $24 \mathrm{~h}$. Cells were harvested with a kinase buffer. The immunoprecipitated kinase assay for cyclin D1-CDK4 was performed as described in Materials and methods. Phosphorylation of $\mathrm{Rb}-\mathrm{C}$ fusion protein was detected by western blot analysis. NC, the negative control without immunoprecipitated cyclin D1. (C) Effect of the DNAzyme on pRb-E2F pathway in CNE1 and CNE1-LMP1 cells. CNE1 and CNE1-LMP1 cells grown in 6-well plates were transfected with DZ1 or control ODN $(2 \mu \mathrm{M})$ for $24 \mathrm{~h}$. Total cell lysates were analyzed by western blotting using specific antibodies, respectively. 

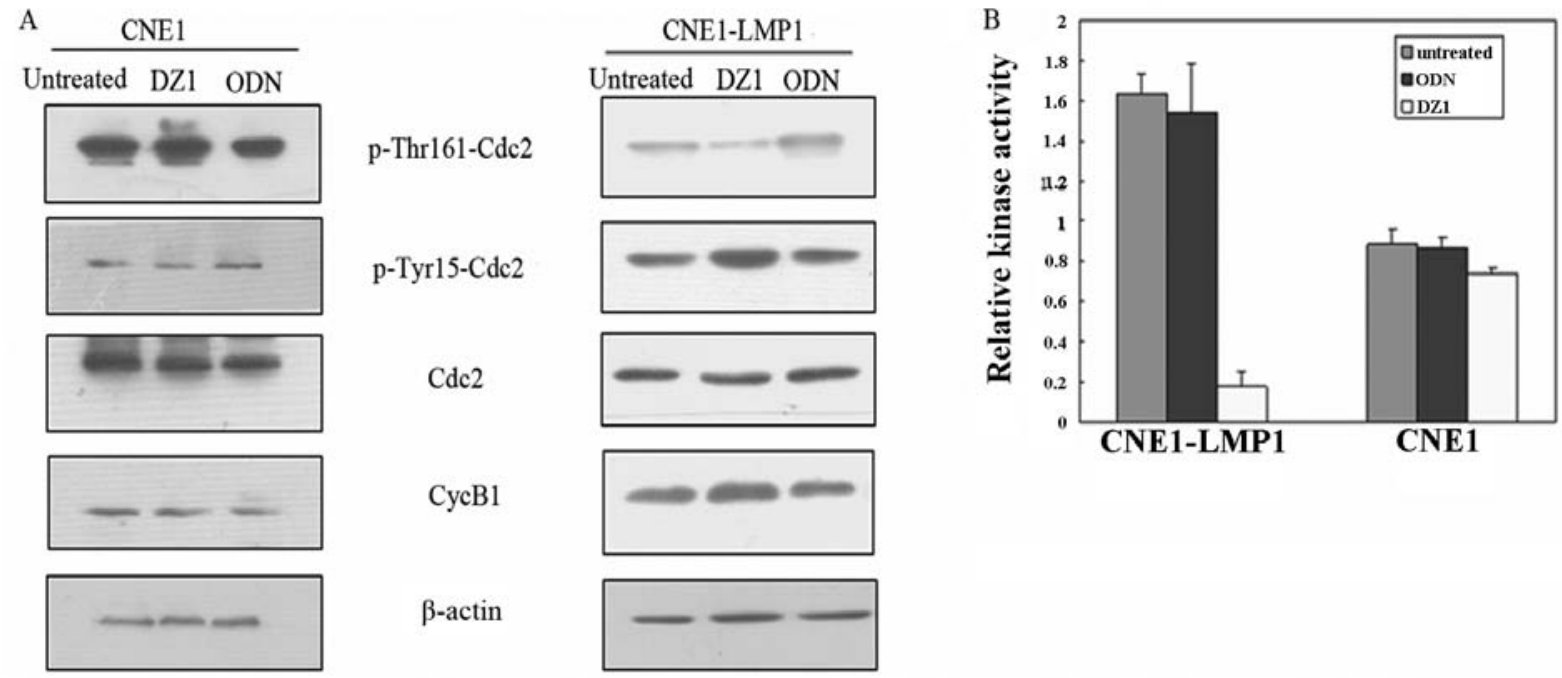

Figure 5. DZ1 suppression of the cdc2 kinase activity in CNE1-LMP1 cells. (A) Western blot analysis of G2/M cell cycle regulators cyclin B1 and cdc2 in CNE1-LMP1 cells. (B) ELISA assay of cdc2 kinase activity of CNE1, and CNE1-LMP1 cells ( $\mathrm{n}=3$ ).

In $\mathrm{pRb}$, the Ser780 site is specifically phosphorylated by the cyclin D/CDK4 complex both in vivo and in vitro (29). Having shown that the downregulation of LMP1 expression by DZ1 lessened the CDK4/cyclin D1 complex, we next examined if the activity of the CDK4/cyclin D1 complex was affected in the context of $\mathrm{pRb}$ phosphorylation. Using the Immunoprecipitation Kinase Assay, it was shown that the downregulation of the LMP1 expression by DZ1 led to a significant reduction of the phosphorylation of Ser780 in pRb (Fig. 4B). The result indicated that the LMP1-specific DNAzyme 1 could slow down the G1 to $\mathrm{S}$ transition through the suppression of activation of cyclin D1-CDK4.

The CDK4/cyclin D1 complex phosphorylates pRb that transcriptionally activates some important transcriptional factors such as E2Fs, which promotes the expression of the downstream proteins such as cyclin A. Entry into $S$ phase is controlled, in part, by the level of active E2F (30). To explore whether LMP1 could regulate the pRb signaling pathway, we investigated the change of phosphorylation status of pRb induced by LMP1-specific DNAzyme 1 and the level of the pRb-regulated E2F. As shown in Fig. 4C, DZ1 mediated suppression of LMP1 could markedly decrease the level of phosphorylated $\mathrm{pRb}$, while total $\mathrm{pRb}$ level remained unchanged. This decreased level of pRb phosphorylation directly impacted on the E2F1 expression.

EBV-LMPI-targeted DNAzyme 1 does not affect expression of $c d c 2$, but downregulates levels of phosphorylation of $c d c 2$ on Thr161 and cdc2 kinase activity. Cdc2 is a critical regulator during the $\mathrm{G} 2$ phase progression. Its activation depends on the Thr161 phosphorylation and dephosphorylation of the Thr14 and Tyr15 (31,32). Thus, the status of Thr161 phosphorylation of cdc2 partially reflects cdc2 kinase activity. To further examine whether the G2/M checkpoint could contribute to the decrease of the number of cells in G2/M phase, cdc2 kinase assay was performed.

The results showed that the level of phosphorylation of the Thr161 was decreased and the dephosphorylation of Tyr15 was increased in DZ1 treated CNE1-LMP1 cells, whereas ODN had no effect (Fig. 5A). There was no difference in the level of cdc2 and cyclin B1 expression in CNE1-LMP1 cells with or without DZ1 treatment. ELISA results demonstrated that cdc2 kinase activity in untreated CNE1-LMP1 cells was nearly 5-fold higher than that in the cells treated with DZ1. Together the LMP1-specific DNAzyme 1 significantly suppressed the LMP1 expression and attenuated cdc2 kinase activity (Fig. 5B).

\section{Discussion}

LMP1, as a key oncogenic protein in NPC, plays important roles in several signal transduction pathways that are involved in cell proliferation, oncogenesis and apoptosis $(33,34)$. There are some reports showing that the silencing of EBV-LMP1 by siRNA slowed the cell cycle progression and enhance chemosensitivity (35). Here we show that EBV-LMP1 may through two cell cycle checkpoints, regulate the cell cycle distribution affecting the DNA damage response. This was evidenced by the present data showing that the LMP1-targeted DNAzyme 1 induced cell accumulation in G1 phase and decreased the number of cells in $\mathrm{G} 2 / \mathrm{M}$ phase. H2AX is one of several genes coding for histone H2A. H2AX becomes phosphorylated on Ser139, then called $\gamma-\mathrm{H} 2 \mathrm{AX}$, as a reaction on DNA double-strand breaks (DSB). The modification can happen in the response to ionizing radiation. As a sensitive target for looking at DSBs in cells, $\gamma-\mathrm{H} 2 \mathrm{AX}$ is considered as a DNA damage marker. We found that the DNA damage marker $\gamma-\mathrm{H} 2 \mathrm{AX}$ was significantly increased by DZ1 in CNE1-LMP1 cells shown by western blot and immunofluorescent analyses. The DNA damage response factor p-p53 was downregulated by DZ1 and the proliferation of LMP1-positive cells was also inhibited by DZ1. Taken together silencing of EBV-LMP1 induced DNA damage and caused cell cycle arrest in LMP1 positive cells.

Cell cycle change is sometimes controlled by the cycling checkpoints. We found downregulation of LMP1 could inhibit expression of cyclin D1 and CDK4 as well as cyclin E and CDK2; suppress cyclin D1 and CDK4 interaction and the 
activity of the complex; and decrease the $\mathrm{Rb}$ protein phosphorylation at Ser780 site. E2F1 is an important regulator of cell entry to $S$ phase (36). Our results showed the DZ1 could suppress the $\mathrm{Rb} / \mathrm{E} 2 \mathrm{~F} 1$ pathway and markedly downregulate the expression of E2F1. In addition, the DZ1-mediated suppression of LMP1 could markedly decrease the cdc2 kinase activity. Taken together, our data suggested that the G1 phase arrest caused by the LMP1 targeted DNAzyme 1 is likely through the G1/S and G2/M checkpoints.

In NPC cells, it has been shown that LMP1 impacted on cell proliferation and growth at three levels: stimulation of quiescent cells to re-enter the cell cycle, acceleration of cycling cells through the G1 phase and increase of cycling cells in the $\mathrm{G} 2 / \mathrm{M}$ phases $(37,38)$. In our previous study we demonstrated that LMP1 expression could enhance phosphorylation of p105-Rb to upregulate E2F1 expression and increase E2F1 ability to transactivate the downstream signal molecules through inhibiting p16INK4A expression. In the present study, we provided further evidence showing that LMP1 plays critical roles in controlling the cell cycle. First, downregulation of the expression of LMP1 could decrease the expression of the G1/S related molecules and disrupt their interactions and activities, which consequentially suppressed the $\mathrm{pRb}$-E2F pathway leading to cell cycle arrest through the restriction point in G1 phase. Second, downregulation of LMP1 could inhibit the cdc2 kinase activity, which led to cell cycle arrest through the restriction point in G2 phase. Therefore, LMP1 could be a central regulator in oncogenesis of EBV-positive cancers, which further support the notion that LMP1 could potentially be an important therapeutic target for NPC.

It has been well established that different types of cells underwent apoptosis from different cell cycle stages under different stimulus $(39,40)$. For example, in HeLa cells, UV caused apoptosis from G1 phase, while the camptothecin (CPT) induced apoptosis from $S$ phase. Several other previously reported cancer-preventive agents seem to act in a manner similar to LMP1-targeted DNAzyme 1. For example, dactylone and inositol hexaphosphate were reported to decrease CDK4 and cyclin D1 protein levels and also showed an inhibitory effect on Rb phosphorylation at Ser780, Ser807 and Ser811, causing G1 arrest and apoptotic death of human prostate carcinoma LNCaP cells (41). In a previous study, we reported that the LMP1 targeted DNAzymes in combination with radiation could enhance radiosensitivity to NPC both in vitro and in vivo $(22,42)$. Thus, the $\mathrm{G} 1$ phase arrest induced by DNAzyme accompanied by DNA damage may cause the NPC cells to enter apoptosis.

In conclusion, LMP1 plays an important role in oncogenesis of the EBV-positive cancers. We presented data suggesting that downregulation of EBV-LMP1 by DNAzyme 1 induced DNA damage leading to G1 phase arrest. Our data imply that the G1 phase arrest caused by the LMP1 targeted DNAzyme 1 is likely through the G1/S and G2/M checkpoints. The biological significance of G1 phase arrest could relate to the sensitization of the EBV-positive cancer cells to either radiotherapy or chemotherapy. Thus, targeting LMP1 by DNAzyme might present a novel strategy to sensitize the EBV-LMP1-positive cells to improve the current treatment efficacy.

\section{Acknowledgements}

This study was supported by the National Natural Science Foundation of China (30930101, 81072220 and 81201171), the National Basic Research Program of China (2009CB521801 and 2011CB504300), and the Fundamental Research Funds for the Central Universities.

\section{References}

1. Ponce RA,GelzleichterT,Haggerty HG, et al: Immunomodulation and lymphoma in humans. J Immunotoxicol: Jun 7, 2013 (Epub ahead of print).

2. Ghosh SK, Perrine SP, Williams RM and Faller DV: Histone deacetylase inhibitors are potent inducers of gene expression in latent EBV and sensitize lymphoma cells to nucleoside antiviral agents. Blood 119: 1008-1017, 2012.

3. Brocqueville G, Ndour PA, Ouk TS, et al: LMP1-induced cell death may contribute to the emergency of its oncogenic property. PLoS One 8: e60743, 2013.

4. Smuk G, Illes A, Keresztes K, et al: Pheno- and genotypic features of Epstein-Barr virus associated B-cell lymphoproliferations in peripheral T-cell lymphomas. Pathol Oncol Res 16: 377-383, 2010.

5. Diduk SV, Smirnova KV, Pavlish OA and Gurtsevitch VE: Functionally significant mutations in the Epstein-Barr virus LMP1 gene and their role in activation of cell signaling pathways. Biochemistry (Mosc) 73: 1134-1139, 2008.

6. Everly DN Jr, Mainou BA and Raab-Traub N: The ID proteins contribute to the growth of rodent fibroblasts during LMP1-mediated transformation. Virology 376: 258-269, 2008.

7. Wu ZZ, Chow KP, Kuo TC, Chang YS and Chao CC: Latent membrane protein 1 of Epstein-Barr virus sensitizes cancer cells to cisplatin by enhancing NF-kappaB p50 homodimer formation and downregulating NAPA expression. Biochem Pharmacol 82: 1860-1872, 2011.

8. Lu JH, Tang YL, Yu HB, et al: Epstein-Barr virus facilitates the malignant potential of immortalized epithelial cells: from latent genome to viral production and maintenance. Lab Invest 90: 196-209, 2010

9. Gan R, Xie X, He J, et al: Gene analysis of Epstein-Barr virus-associated lymphomas in $\mathrm{Hu}-\mathrm{Pbl} / \mathrm{SCID}$ chimeras. Tumori 96: 465-472, 2010

10. Arcipowski KM and Bishop GA: TRAF binding is required for a distinct subset of in vivo B cell functions of the oncoprotein LMP1. J Immunol 189: 5165-5170, 2012.

11. Yoshizaki T, Kondo S, Wakisaka N, et al: Pathogenic role of Epstein-Barr virus latent membrane protein-1 in the development of nasopharyngeal carcinoma. Cancer Lett 337: 1-7, 2013.

12. Zheng H, Li LL, Hu DS, Deng XY and Cao Y: Role of Epstein-Barr virus encoded latent membrane protein 1 in the carcinogenesis of nasopharyngeal carcinoma. Cell Mol Immunol 4: 185-196, 2007.

13. Tao YG, Tan YN, Liu YP, et al: Epstein-Barr virus latent membrane protein 1 modulates epidermal growth factor receptor promoter activity in a nuclear factor kappa B-dependent manner. Cell Signal 16: 781-790, 2004.

14. Wang Z, Luo F, Li L, et al: STAT3 activation induced by Epstein-Barr virus latent membrane protein1 causes vascular endothelial growth factor expression and cellular invasiveness via JAK3 And ERK signaling. Eur J Cancer 46: 2996-3006, 2010.

15. Horikawa T, Yang J, Kondo S, et al: Twist and epithelialmesenchymal transition are induced by the EBV oncoprotein latent membrane protein 1 and are associated with metastatic nasopharyngeal carcinoma. Cancer Res 67: 1970-1978, 2007.

16. Dawson CW, Laverick L, Morris MA, Tramoutanis G and Young LS: Epstein-Barr virus-encoded LMP1 regulates epithelial cell motility and invasion via the ERK-MAPK pathway. J Virol 82: 3654-3664, 2008.

17. Sheu LF, Chen A, Wei YH, et al: Epstein-Barr virus LMP1 modulates the malignant potential of gastric carcinoma cells involving apoptosis. Am J Pathol 152: 63-74, 1998.

18. Li G, Li XP, Peng Y, Liu X and Li XH: Effect of inhibition of EBV-encoded latent membrane protein-1 by small interfering RNA on EBV-positive nasopharyngeal carcinoma cell growth. Di Yi Jun Yi Da Xue Xue Bao 24: 241-246, 2004. 
19. Li XP, Li G, Peng Y, Kung HF and Lin MC: Suppression of Epstein-Barr virus-encoded latent membrane protein-1 by RNA interference inhibits the metastatic potential of nasopharyngeal carcinoma cells. Biochem Biophys Res Commun 315: 212-218, 2004.

20. Ndour PA, Brocqueville G, Ouk TS, et al: Inhibition of latent membrane protein 1 impairs the growth and tumorigenesis of latency II Epstein-Barr virus-transformed T cells. J Virol 86: 3934-3943, 2012.

21. Yang L, Lu Z, Ma X, Cao Y and Sun LQ: A therapeutic approach to nasopharyngeal carcinomas by DNAzymes targeting EBV LMP-1 gene. Molecules 15: 6127-6139, 2010.

22. Lu ZX, Ma XQ, Yang LF, et al: DNAzymes targeted to EBV-encoded latent membrane protein-1 induce apoptosis and enhance radiosensitivity in nasopharyngeal carcinoma. Cancer Lett 265: 226-238, 2008.

23. Glaser R, Zhang HY, Yao KT, et al: Two epithelial tumor cell lines (HNE-1 and HONE-1) latently infected with Epstein-Barr virus that were derived from nasopharyngeal carcinomas. Proc Natl Acad Sci USA 86: 9524-9528, 1989.

24. Zhao Y, Tao YG, Luo FJ, Tang FQ, Tang M and Cao Y: Interference effect of epigallocatechin-3-gallate on targets of nuclear factor kappaB signal transduction pathways activated by EB virus encoded latent membrane protein 1. Int J Biochem Cell Biol 36: 1473-1481, 2004.

25. Benimetskaya L, Takle GB, Vilenchik M, Lebedeva I, Miller P and Stein CA: Cationic porphyrins: novel delivery vehicles for antisense oligodeoxynucleotides. Nucleic Acids Res 26 : 5310-5317, 1998

26. Lu ZX, Ye M, Yan GR, et al: Effect of EBV LMP1 targeted DNAzymes on cell proliferation and apoptosis. Cancer Gene Ther 12: 647-654, 2005.

27. Sugimoto M, Martin N, Wilks DP, et al: Activation of cyclin D1-kinase in murine fibroblasts lacking both p21(Cip1) and p27(Kip1). Oncogene 21: 8067-8074, 2002.

28. Ling S and Lin WC: EDD inhibits ATM-mediated phosphorylation of p53. J Biol Chem 286: 14972-14982, 2011.

29. Kitagawa M, Higashi H, Jung HK, et al: The consensus motif for phosphorylation by cyclin D1-Cdk4 is different from that for phosphorylation by cyclin A/E-Cdk2. EMBO J 15: 7060-7069, 1996.

30. Hansen U, Owens L and Saxena UH: Transcription factors LSF and E2Fs: tandem cyclists driving G0 to S? Cell Cycle 8: 2146-2151, 2009.
31. Zhang XH, Zou ZQ, Xu CW, Shen YZ and Li D: Myricetin induces $\mathrm{G} 2 / \mathrm{M}$ phase arrest in HepG2 cells by inhibiting the activity of the cyclin B/Cdc2 complex. Mol Med Rep 4: 273-277, 2011.

32. Tan M, Jing T, Lan KH, et al: Phosphorylation on tyrosine-15 of p34(Cdc2) by ErbB2 inhibits p34(Cdc2) activation and is involved in resistance to taxol-induced apoptosis. Mol Cell 9: 993-1004, 2002.

33. Dawson CW, Port RJ and Young LS: The role of the EBV-encoded latent membrane proteins LMP1 and LMP2 in the pathogenesis of nasopharyngeal carcinoma (NPC). Semin Cancer Biol 22: 144-153, 2012.

34. Tulalamba $\mathrm{W}$ and Janvilisri T: Nasopharyngeal carcinoma signaling pathway: an update on molecular biomarkers. Int J Cell Biol 2012: 594681, 2012.

35. Mei YP, Zhou JM, Wang Y, et al: Silencing of LMP1 induces cell cycle arrest and enhances chemosensitivity through inhibition of AKT signaling pathway in EBV-positive nasopharyngeal carcinoma cells. Cell Cycle 6: 1379-1385, 2007.

36. Korotayev K, Chaussepied M and Ginsberg D: ERK activation is regulated by E2F1 and is essential for E2F1-induced S phase entry. Cell Signal 20: 1221-1226, 2008.

37. Deng L, Yang J, Zhao XR, et al: Cells in G2/M phase increased in human nasopharyngeal carcinoma cell line by EBV-LMP1 through activation of NF-kappaB and AP-1. Cell Res 13: 187-194, 2003.

38. Zhao XR, Gu HH, Weng XX, Yi W, Deng XY and Cao Y: The primary study on expression and function of D-type cyclins in nasopharyngeal carcinoma cell lines. Sheng Wu Hua Xue Yu Sheng Wu Wu Li Xue Bao (Shanghai) 32: 192-196, 2000.

39. Liu DX and Greene LA: Neuronal apoptosis at the G1/S cell cycle checkpoint. Cell Tissue Res 305: 217-228, 2001.

40. Tao D, Wu J, Feng Y, Qin J, Hu J and Gong J: New method for the analysis of cell cycle-specific apoptosis. Cytometry A 57: 70-74, 2004.

41. Agarwal C, Dhanalakshmi S, Singh RP and Agarwal R: Inositol hexaphosphate inhibits growth and induces G1 arrest and apoptotic death of androgen-dependent human prostate carcinoma LNCaP cells. Neoplasia 6: 646-659, 2004.

42. Ma X, Yang L, Xiao L, et al: Down-regulation of EBV-LMP1 radio-sensitizes nasal pharyngeal carcinoma cells via NF-kappaB regulated ATM expression. PLoS One 6: e24647, 2011. 